\title{
Effect of a "T-Band" Kick Training Protocol on Postural Sway
}

\author{
By: Andrew G. Baker, William G. Webright, and David H. Perrin ${ }^{1}$
}

Baker, A.G., Webright, W.G., Perrin, D.H. (1998). Effect of a "T-band" kick training protocol on postural sway. Journal of Sport Rehabilitation, 7:122-127.

\section{***Note: Figures may be missing from this format of the document}

\begin{abstract}
:
The purpose of this study was to examine the effects of a resistive tubing kick training protocol on postural sway in uninjured collegiate wrestlers. An experimental group $(\mathrm{n}=10)$ performed a progressive resistive tubing kick training protocol three times per week for 6 weeks. A control group $(n=9)$ performed no resistive tubing training during the 6 weeks. Postural sway (stability index) was assessed before and after the 6-week training period. ANOVAs demonstrated no significant interactions, although significant main effects were found for group and eye condition. The experimental group demonstrated less postural sway than the control group regardless of training, and postural sway was greater with the eyes closed than with the eyes open. Resistive tubing kick training does not significantly improve postural sway in healthy collegiate wrestlers. Further research should examine the potential benefits of proprioceptive training using a greater intensity of training and/or using subjects who have a greater potential for improvement.

\section{Article:}

Proprioceptive exercises are commonly used in treating lower extremity injuries such as knee and ankle sprains. Repeated stresses on these joints can result in ligamentous laxity and proprioceptive impairment $(1,4,11)$. This impairment causes inadequate feedback to the brain, which could increase the risk of injury. The use of proprioceptive rehabilitation exercises is essential to increase proprioceptive feedback to the brain. Many authors have investigated the effects of proprioceptive rehabilitation on the lower extremity $(2,3,5,9,11)$, but none have investigated the effect of resistive exercise on postural sway.
\end{abstract}

The terms related to proprioception and postural sway as used in the literature are confusing (7). Proprioception has been defined as "the ability to receive input from muscles, tendons, and joints and to process that information in a meaningful way in the central nervous system" (6, p. 72) Proprioceptive feedback to the brain contributes to the body's ability to maintain postural stability.

Many proprioception exercises are used in clinical settings. The simplest is a modified Romberg test (stork stand) position in which the subject stands on the injured leg and balances for a specified duration. The stork stand can be performed initially with eyes open and then progress

\footnotetext{
${ }^{1}$ Andrew G. Baker is with HealthSouth Sports Medicine in Roseville, MN, and was a master's student at the University of Virginia, Charlottesville, VA, at the time of this study. William G. Webright is a doctoral candidate in sports medicine at the University of Virginia. David H. Perrin is with the Department of Human Services, Curry School of Education, University of Virginia. Direct correspondence to David H. Perrin, University of Virginia, Memorial Gymnasium, Charlottesville, VA 22903.
} 
to eyes closed on stable or unstable surfaces. Balance boards consisting of platforms with uniplanar or spherical attachments are also commonly used. Tomaszewski (8) advocated the use of a T- band in proprioceptive rehabilitation to stimulate neuromuscular activity and increase ankle stability. Subjects stood balanced on the injured limb and performed kicking motions with the uninjured extremity against T-band resistance. The kicking motion was used to stimulate proprioceptors around the injured extremity in response to positional changes of the body's center of gravity (11).

No research is currently available regarding the use of resistive tubing and its effect on postural sway. The purpose of this study was to determine the effects of resistive tubing training on postural sway. It was our hypothesis that postural sway would decrease following a 6-week resistive tubing kick training program that challenged unilateral standing balance.

\section{METHODS}

Subjects

Nineteen NCAA Division I wrestlers (age $=19.7 \pm 1.1$ years, height $=1.75 \pm 0.07 \mathrm{~m}$, weight $=$ $75.7 \pm 8.8 \mathrm{~kg}$ ) with no lower extremity injury (past 6 months), vestibular problems, or experience with postural stability testing were randomly assigned to one of two groups. The experimental group $(n=10)$ performed a progressive resistive tubing kick training protocol three times per week for 6 weeks. The control group $(n=9)$ performed no resistive tubing training during the 6 week period. Prior to participation, all subjects read and signed an informed consent agreement approved by the university review board.

\section{Procedure}

Postural sway was measured on the Biodex Stability System (Biodex Medical Systems, Shirley, NY). Stability index (SI) is the variance of foot platform displacement in degrees, which was the dependent measure of postural sway. The Biodex Stability System permits a maximum of $20^{\circ}$ of platform displacement in all directions. Overall SI represents platform motion in all planes, anterior/posterior SI represents motion in the sagittal plane, and medial/lateral SI represents frontal plane motion.

Subjects were tested barefoot in a unilateral stance (Figure 1). Testing was performed within 1 week prior to and after the 6-week training protocol. Extremity (right and left) and eye (open and closed) conditions were tested in a counter- 


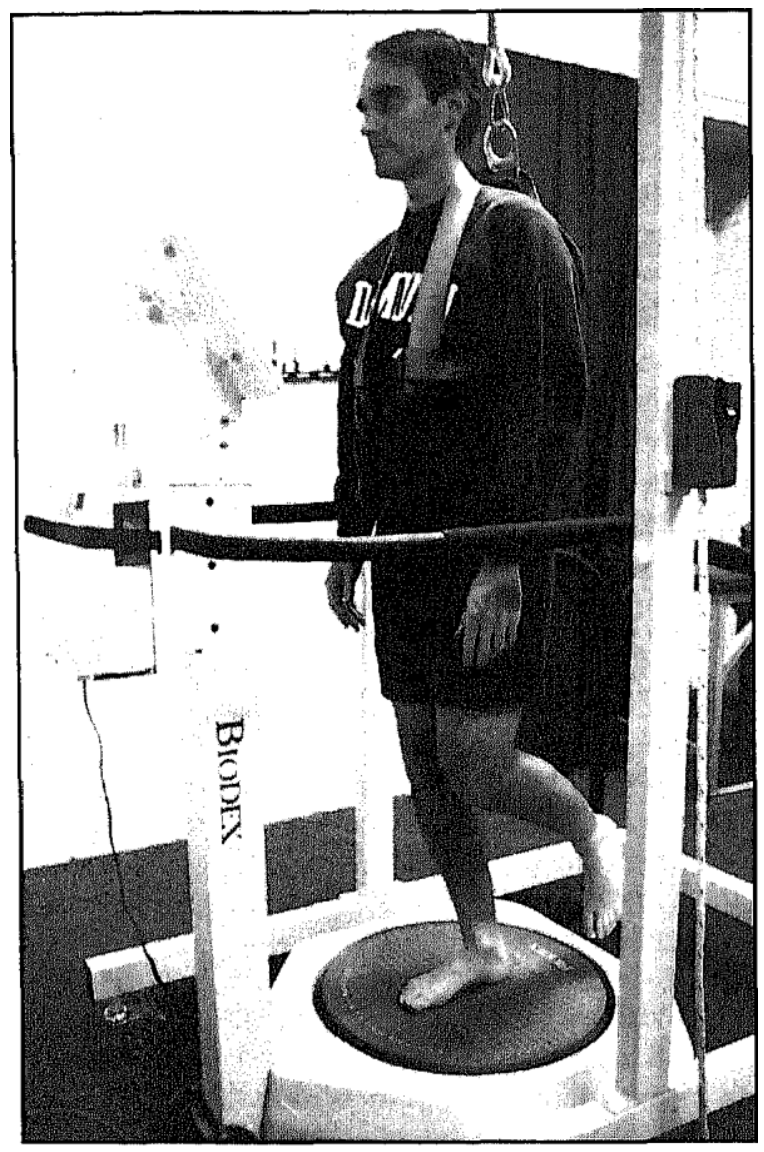

Figure 1- Postural sway testing.

balanced order. The foot of the test extremity was positioned on the center of the stability platform with the knee slightly flexed. The contralateral extremity was positioned according to subject comfort but was not placed on top of or hooked behind the test leg. Arm position was not controlled. A harness was used as a safety precaution but did not impede body sway. Subjects stood on both legs between tests. Each test lasted $1 \mathrm{~min}$, with the platform becoming progressively less stable. Platform stability began at Level 6 (most stable) and decreased one level every $10 \mathrm{~s}$ until it reached Level 1 (least stable).

Resistive exercises were performed with Esmarch wrap tubing from MedSurg Isolyser (Herndon, VA). The 3 in. wide by 3 yard long tubing was securely fastened to an immovable object. We controlled resistance by instructing subjects to remove any slack in the tubing by moving away from where the tubing was fastened. Subjects, who were barefoot, placed the left ankle through a loop at the unfastened end of the tubing. Exercise consisted of short, quick kicking motions performed in hip flexion, extension, abduction, and adduction with the knee extended. The exercises were used to perturb single-leg balance of the right lower extremity (Figure 2). Table 1 depicts the exercise progression.

\section{Data Analysis}

A three within (test, extremity, eyes) and one between (group) repeated-measures analysis of variance was performed to analyze the stability index data. Probability level was set at $\mathrm{p}<.05$. 
Tukey's Honestly Significant Difference post hoc testing was used to determine where significant differences occurred.

\section{RESULTS AND DISCUSSION}

The means and standard deviations for stability index are presented in Table 2. No significant interactions were found. Significant main effects were found for group,

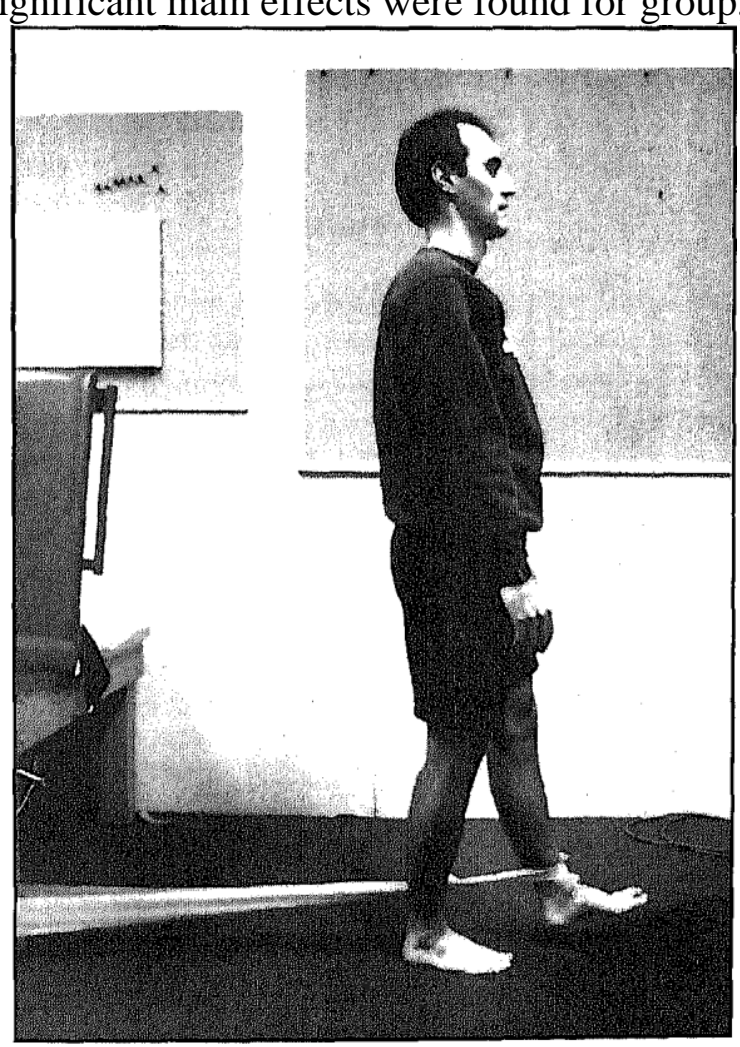

Figure 2 - Resistive tubing kick training. 
Table 1 Progressive Training Protocol

\begin{tabular}{clc}
\hline Week & Eyes & Repetitions \\
\hline 1 & Open & 30 \\
2 & Closed & 30 \\
3 & Open & 40 \\
4 & Closed & 40 \\
5 & Open & 50 \\
6 & Closed & 50 \\
\hline
\end{tabular}

"Repetitions performed in four directions: hip flexion, hip extension, abduction, adduction.

Table 2 Stability Index (degrees)

\begin{tabular}{lrrrr}
\hline & \multicolumn{2}{c}{ Control } & \multicolumn{2}{c}{ Experimental } \\
& $M$ & $S D$ & $M$ & $S D$ \\
\hline Pre / RL / EO & 4.69 & 1.86 & 4.24 & 1.16 \\
Post / RL / EO & 4.60 & 1.49 & 3.44 & 1.00 \\
Pre / RL / EC & 10.12 & 2.71 & 9.11 & 2.44 \\
Post / RL / EC & 9.84 & 2.37 & 7.62 & 2.27 \\
Pre / LL / EO & 3.89 & 1.54 & 3.40 & 1.17 \\
Post / LL / EO & 4.33 & 1.53 & 3.33 & 1.06 \\
Pre / LL / EC & 10.23 & 1.53 & 9.50 & 2.04 \\
Post / LL / EC & 10.03 & 1.62 & 8.27 & 2.36 \\
& & & & \\
\hline
\end{tabular}

Note. Pre $=$ pretest post $=$ posttest RL = right leg; $\mathrm{LL}=$ left leg; $\mathrm{EC})=$ eyes open; $\mathrm{EC}=$ eyes closed.

$\mathrm{F}(1,17)=4.43, \mathrm{p}<.05$, and eye condition, $\mathrm{F}(1,17)=245.41, \mathrm{p}<.05$. The experimental group had less postural sway than the control group, and postural sway was greater with the eyes closed than open.

The primary finding was that the resistive tubing training protocol did not significantly affect postural sway. We rejected our hypothesis that a proprioceptive rehabilitation program using resistive tubing kick training would decrease the amount of postural sway of the right leg.

Examination of the pre- and posttest mean scores for both groups suggests a trend toward a training effect (with exception of the control/left leg/eyes open condition). Both groups improved their pre- to posttest scores. The experimental group appeared to have a greater improvement of stability index with eyes open $\left(4.24\right.$ to $\left.3.44^{\circ}\right)$ and eyes closed $\left(9.11\right.$ to $\left.7.62^{\circ}\right)$ compared to the control group with eyes open $\left(4.69\right.$ to $\left.4.60^{\circ}\right)$ and eyes closed $\left(10.12\right.$ to $\left.9.84^{\circ}\right)$. This trend provides an incentive for further research using a larger sample size. Because some subjects failed to meet participation criteria and there was some attrition of athletes during the season, statistical power was reduced from a predetermined ideal using 32 subjects to the resultant 19 subjects. 
Another limitation of this study was the use of elite athletes for training purposes. Collegiate wrestlers are elite athletes with high levels of fitness in a sport that frequently challenges postural stability. Our subjects' probable high level of postural stability may have limited our attempt to induce a training effect. Future research should consider using untrained or rehabilitating injured subjects.

The lack of improvement in our study may also have been due to the brevity of training frequency and duration. Our exercise protocol consisted of training three times per week for 6 weeks. Cox et al. (2) used a three times per week, 4-week training protocol and found no differences in postural stability as measured by sway index on the Chattecx Balance System. Hoffman and Payne (5) used a three times per week, 10-week protocol involving a BAPS board and found significant differences in sway variability using a Kistler force platform. Future research should examine the potential benefits of proprioception training using a greater intensity of training, or using a T-band in conjunction with other closed chain exercises.

\section{REFERENCES}

1. Cailliet, R. Foot and Ankle Pain (2nd ed.). Philadelphia: Davis, 1983, pp. 148-158.

2. Cox, ED., S.M. Lephart, and J.J. Irrgang. Unilateral balance training of noninjured individuals and the effects on postural sway. J. Sport Rehabil. 2:87-96, 1993.

3. Freeman, M.A.R. Co-ordination exercises in the treatment of functional instability of the foot. Physiotherapy 51:393-395, 1965.

4. Freeman, M.A.R., M.R.E. Dean, and I.W.F. Hanham. The etiology and prevention of functional instability of the foot. J. Bone Joint Surg. (Br.) 47B:678-685, 1965.

5. Hoffman, M., and V.G. Payne. The effects of proprioceptive ankle disk training on healthy subjects. J. Orthop. Sports Phys. Ther. 21:90-93, 1995.

6. Irrgang, J.J., S.L. Whitney, and E.D. Cox. Balance and proprioceptive training for rehabilitation of the lower extremity. J. Sport Rehabil. 3:68-83, 1994.

7. Mattacola, C.G., D.A. Lebsack, and D.H. Perrin. Intertester reliability of assessing postural sway using the Chattecx Balance System. J. Athletic Training 30:237-242, 1995.

8. Tomaszewski, D. T-band kicks ankle proprioception program. Athletic Training, JNATA. 26:216-218, 1991.

9. Tropp, H., C. Askling, and J. Gillquist. Prevention of ankle sprains. Am. J. Sports Med. 13:259-262, 1985.

10. Tropp, H., J. Ekstrand, and J. Gillquist. Factors affecting stabilometry recordings of single limb stance. Am. J. Sports Med. 12:185-188, 1984.

11. Wilkerson, G.B., and A.J. Nitz. Dynamic ankle stability: Mechanical and neuromuscular interrelationships. J. Sport Rehabil. 3:43-57, 1994. 